\title{
Erratum to: Association of Mycobacterium avium subspecies paratuberculosis infection with milk production and calving interval in Iranian Holsteins
}

Maryam Ansari-Lari • Masoud Haghkhah • Fereshteh Mahmoodi

Published online: 2 May 2012

(C) Springer Science+Business Media B.V. 2012

Erratum to: Trop Anim Health Prod

DOI 10.1007/s11250-011-0047-3

In the tables 2, 4 and 5, the category "Parity 4" should be added as follows.

LJSH Suggestion for the erratum

In tables 2, 4 and 5 . The parity column should read $1,2,3,4$ not $1,2,3,5$.

The table captions should all have an additional sentence as follows:

'Data for parity 5 was used as the baseline for calculating the information for Parities 1-4)

Tables 2, 4 and 5

The online version of the original article can be found at http://dx.doi. org/10.1007/s11250-011-0047-3.

M. Ansari-Lari $(\bowtie)$

Department of Food Hygiene and Public Health, Shiraz University,

Shiraz 71345, Iran

e-mail: ansari@shirazu.ac.ir

M. Haghkhah · F. Mahmoodi

Department of Pathobiology, School of Veterinary Medicine,

Shiraz University,

Shiraz 71345, Iran 
Table 2 Effect of infection with Mycobacterium paratuberculosis on 305-day milk production in 21 dairy herds, Shiraz, Iran; comparison of positive cows with negative cows

\begin{tabular}{lcrrc}
\hline Effect & Estimate & \multicolumn{1}{l}{ SE } & $t$ Value & $P$ value \\
\hline Intercept & $6,263.14$ & 603.11 & 10.38 & $<0.001$ \\
Positive cows $^{\mathrm{a}}$ & & & & \\
Negative cows & $1,009.24$ & 382.61 & 2.64 & 0.009 \\
Lactation length & 2.88 & 0.59 & 4.87 & $<0.001$ \\
Genetic purity & 6.45 & 4.17 & 1.55 & 0.12 \\
Parity 1 & $-1,592.6$ & 292.19 & -5.45 & $<0.001$ \\
Parity 2 & -572.9 & 285.32 & -2.01 & $<0.001$ \\
Parity 3 & -132 & 295.77 & -0.45 & 0.65 \\
Parity 4 & -60.80 & 315.44 & -0.19 & 0.84 \\
\hline
\end{tabular}

$S E$ standard error

${ }^{\text {a }}$ Reference category - 'Data for parity 5 was used as the baseline for calculating the information for Parities 1-4)
Table 5 Effect of Mycobacterium paratuberculosis infection on calving interval, in 21 dairy herds, Shiraz, Iran; comparison of cows from positive herds with cows from negative herds

\begin{tabular}{lcccc}
\hline Effect & Estimate & SE & $t$ Value & $P$ value \\
\hline Intercept & 339 & 72.18 & 4.70 & $<0.001$ \\
Cows from positive herds ${ }^{\mathrm{a}}$ & & & & \\
Cows from negative herds & -42.13 & 21.84 & -1.93 & 0.058 \\
Lactation length & 0.03 & 0.10 & 0.27 & 0.78 \\
Genetic purity & -0.80 & 0.52 & -1.53 & 0.13 \\
305-day milk & 0.02 & 0.007 & 2.62 & 0.01 \\
Parity 2 & 45.28 & 35.92 & 1.26 & 0.21 \\
Parity 3 & 37.87 & 38.94 & 0.97 & 0.33 \\
Parity 4 & 50.58 & 42.93 & 1.18 & 0.24 \\
\hline
\end{tabular}

$S E$ standard error

${ }^{a}$ Reference category - 'Data for parity 5 was used as the baseline for calculating the information for Parities 1-4)
Table 4 Effect of infection with Mycobacterium paratuberculosis on 305-day milk production in 21 dairy herds, Shiraz, Iran; comparison of cows from positive herds with cows from negative herds

\begin{tabular}{lcrrc}
\hline Effect & Estimate & SE & $t$ Value & $P$ value \\
\hline Intercept & $6,868.11$ & 472.37 & 14.54 & $<0.001$ \\
Cows from positive herds & & & & \\
Cows from negative herds & 509.5 & 178.62 & 2.85 & 0.005 \\
Lactation length & 2.65 & 0.58 & 4.54 & $<0.001$ \\
Genetic purity & 6.81 & 4.12 & 1.65 & 0.09 \\
Parity 1 & $-1,568.29$ & 289.43 & -5.42 & $<0.001$ \\
Parity 2 & -561.1 & 282.69 & -1.98 & 0.048 \\
Parity 3 & -135.3 & 282.99 & -0.46 & 0.64 \\
Parity 4 & -73.81 & 312.56 & -0.24 & 0.81 \\
\hline
\end{tabular}

$S E$ standard error

${ }^{\text {a }}$ Reference category - 'Data for parity 5 was used as the baseline for calculating the information for Parities 1-4) 